\title{
Folate receptor- $\alpha$ expression in resectable hepatic colorectal cancer metastases: patterns and significance
}

\author{
Michael D’Angelica ${ }^{1}$, John Ammori ${ }^{1}$, Mithat Gonen ${ }^{2}$, David S Klimstra ${ }^{3}$, Philip S Low ${ }^{4}$, \\ Linda Murphy ${ }^{5}$, Martin R Weiser ${ }^{1}$, Philip B Paty ${ }^{1}$, Yuman Fong ${ }^{1}$, Ronald P DeMatteo ${ }^{1}$, \\ Peter Allen $^{1}$, William R Jarnagin ${ }^{1}$ and Jinru Shia ${ }^{3}$ \\ ${ }^{1}$ Department of Surgery, Memorial Sloan-Kettering Cancer Center, New York, NY, USA; ${ }^{2}$ Department of \\ Biostatistics, Memorial Sloan-Kettering Cancer Center, New York, NY, USA; ${ }^{3}$ Department of Pathology, \\ Memorial Sloan-Kettering Cancer Center, New York, NY, USA; ${ }^{4}$ Department of Chemistry and Weldon School \\ of Biomedical Engineering, Purdue University, West Lafayette, IN, USA and ${ }^{5}$ Department of Biochemistry \\ and Molecular Biology, Mayo Clinic College of Medicine, Rochester, MN, USA
}

Folate receptor alpha (FR $\alpha$ ), encoded by folate receptor 1 (adult) gene, has emerged as a cancer biomarker and potential therapeutic target. In addition, its expression in tumors may offer prognostic information. The aim of this study was to assess the prognostic value of FR $\alpha$ expression and other common molecular markers in resected liver metastases from colorectal cancer. To maximize potential biological differences, we selected two groups of patients with markedly different outcomes as study subjects. Immunohistochemical analysis of FR $\alpha$ expression and other common markers (thymidylate synthase, p53, p27, BCL2, ki67, MLH1, MSH2 and MGMT) on tissue microarrays was carried out on samples from 160 patients; 56 patients survived at least 10 years following liver resection, and 104 died within 2 years of surgery. These markers were evaluated and compared with standard clinical predictors of outcome including a previously validated clinical risk score. Our results showed that in addition to known clinical risk factors, $F R \alpha$ positivity was significantly associated with the early death group $(32 \%$ compared with $13 \% ; P=0.03)$. None of the other common molecular markers were differentially expressed between the two groups. On multivariate analysis, clinical risk score, margin status and $F R \alpha$ expression were independently associated with outcome. Specific multivariate comparisons confirmed that $F R \alpha$ expression was associated with outcome independent of the clinical risk score and margin. These data demonstrate that FR $\alpha$ expression is present in a subset of resected hepatic colorectal cancer metastases, and this marker is independently associated with survival after hepatic resection. The prognostic value of $F R \alpha$ expression and the utility of FR $\alpha$-targeted therapies in stage-IV colorectal cancer patients deserve further exploration.

Modern Pathology (2011) 24, 1221-1228; doi:10.1038/modpathol.2011.82; published online 13 May 2011

Keywords: folate receptor 1 (adult); folate-targeted therapy; liver metastasis

The liver is the most common site of metastasis from colorectal carcinoma. Although 20 to $25 \%$ of colorectal carcinoma patients present with synchronous liver metastases, another $60 \%$ develop such meta-

Correspondence: Dr J Shia, MD, Department of Pathology, Memorial Sloan-Kettering Cancer Center, 1275 York Avenue, New York, NY 10021, USA.

E-mail: Shiaj@mskcc.org

Received 13 January 2011; revised 4 April 2011; accepted 4 April 2011; published online 13 May 2011 stases after the diagnosis of the primary tumor. ${ }^{1,2}$ Surgical resection is potentially curative for colorectal cancer metastases confined to the liver, with a 5-year survival rate between 30 and $50 \% .^{3}$ Some patients, however, recur early after resection and have a poor outcome. A clinical risk score developed by Fong et $a l^{1}$ incorporates five preoperative criteria including nodal status of the primary lesion, disease-free interval, number of hepatic metastases, size of the largest metastasis and preoperative carcinoembryonic antigen (CEA) level. This 
preoperative score has been shown to be predictive of outcome and survival for such patients surgically treated with curative intent.

Over the last two decades, efforts to improve prognostication for colorectal carcinoma patients have been directed towards molecular biomarkers. Encouraging developments in the molecular field include the discovery of the microsatellite instability phenotype as a favorable prognostic marker and the absence of Kras and Braf mutations as predictors of response to EGFR inhibitors. ${ }^{4}$ Achievements thus far, however, are far from meeting the demands for a diverse armamentarium required to overcome the significant heterogeneity of both tumor and patient characteristics.

Folate receptor alpha $(\mathrm{FR} \alpha)$ is a cell membraneanchored protein encoded by the Folate receptor 1 (adult) gene (ie, the FOLR1 gene) in human. It binds and transports folic acid, and has recently emerged as a potential tumor biomarker. ${ }^{5-8}$ Its expression in certain epithelial tumors such as ovarian, breast, endometrial and non-small cell lung carcinoma has been associated with tumor behavior. ${ }^{9-12}$ Importantly, FR $\alpha$ expression is tumor specific, as it is significantly higher in tumor cells than in normal cells. ${ }^{13} \mathrm{FR} \alpha$ also has a high affinity for folic acid; and the high affinity is maintained when folic acid is conjugated with other molecules. Such properties of FR $\alpha$ have allowed developments of selective delivery of folate-linked drugs and anti-FR $\alpha$ antibodies to FR $\alpha$-expressing cancer cells. ${ }^{14,15}$ Several FR $\alpha$-targeted therapeutic agents have entered into the early-phase human clinical trials for treatment of ovarian cancers, and have shown promising results. ${ }^{16-20}$

Data on FR $\alpha$ expression in colorectal carcinomas are limited and FR $\alpha$-targeted approaches have not been investigated in this cancer. In a previous study utilizing tissue microarray and immunohistochemistry, we analyzed the status of FR $\alpha$ expression in primary colorectal cancers. ${ }^{21}$ Our results indicated that a subset of colorectal cancers had positive FR $\alpha$ expression detectable by immunohistochemistry, and the expression of FR $\alpha$ was inversely related to tumor microsatellite instability-high phenotype. On univariate analysis, positive $\mathrm{FR} \alpha$ expression in primary colorectal cancer correlated with a worse 5-year disease-specific survival. In the current study, we extended our study of $F R \alpha$ to patients with resected colorectal cancer liver metastases. The analysis of $\mathrm{FR} \alpha$ was carried out in parallel with other commonly evaluated molecular markers including the tumor suppressor gene products p53, p27 and BCL2; proliferation marker ki67; DNA microsatellite instability-related markers MLH1, MSH2 and MGMT; and fluoropyrimidine-related predictive marker thymidylate synthase. We analyzed these molecular markers in the context of previously established clinical risk factors. Our study objective was to evaluate the prognostic value of $F R \alpha$ and other common molecular markers in resected colorectal cancer liver metastases. Such data may be useful for assessing the potential of FR $\alpha$-targeted therapy in patients with resected colorectal cancer liver metastases.

\section{Materials and methods}

\section{Clinical Information}

This study was approved by the Memorial SloanKettering Cancer Center Institutional Review Board. Study subjects were identified from a prospectively maintained database of patients undergoing liver resection for colorectal cancer liver metastases from 1985 to 1994 . To maximize potential biological and molecular differences among study subjects, two groups of patients with markedly different clinical outcome were chosen: (1) those who survived at least 10 years after surgery, termed 'long-term survivor group'; and (2) those who died within 2 years of resection, termed 'early death group'.

Clinical variables were collected from a prospective clinical database and supplemented with review of medical records, operative reports, anesthesia reports and pathology reports as needed. The variables analyzed were as follows: patient demographics, extent of liver resection, bilateral resection, diseasefree interval before hepatic surgery, lymph-node status of the primary tumor, size and number of hepatic metastases, pre-hepatectomy CEA level and the status of the hepatic resection margin. The clinical risk score, as defined previously, was evaluated. ${ }^{1}$ Briefly, a point was assessed for each of the following five factors: (1) disease-free interval less than 12 months, (2) node-positive primary colorectal cancer, (3) greater than one tumor, (4) size of largest tumor greater than $5 \mathrm{~cm}$ and (5) pre-hepatectomy CEA $>200 \mathrm{ng} / \mathrm{ml}$.

\section{Pathological Assessment}

All liver metastasis specimens were analyzed for histology type, number of tumor nodules, size of the largest nodule and margin status. Representative tumor blocks were selected from each case and used for the construction of tissue microarrays. The ATA27 automated arrayer (Beecher Instruments) was used to create the microarrays. The punch size was set at $0.6 \mathrm{~mm}$. Three cores of different areas of the tumor were sampled from each tumor specimen.

Immunohistochemical staining was performed on the tissue microarray slides. The sections were deparaffinized, rehydrated in graded alcohols and processed using the avidin-biotin immunoperoxidase method. Briefly, sections were submitted to antigen retrieval by heat treatment for $15 \mathrm{~min}$ in $0.01 \mathrm{mM}$-citrate buffer at $\mathrm{pH} 6.0$ (For FR $\alpha$ staining, slides were placed in a preheated DAKO Target Retrieval Buffer (DAKO Cytomation, Carpenteria, CA, USA) for 40 min using the ISOTEMP 205 water 
bath (Fisher Scientific)). For Ki-67 antibody, an additional step of incubation in preheated $0.05 \%$ trypsin, $0.05 \% \mathrm{CaCl}_{2}$ in Tris-HCl (pH 7.6) for $5 \mathrm{~min}$ at $37^{\circ} \mathrm{C}$ before microwave treatment was carried out. Slides were subsequently incubated in $10 \%$ normal horse serum for $30 \mathrm{~min}$. The slides were then incubated in appropriately diluted primary antibody. Mouse antihuman monoclonal antibodies to $\mathrm{FR} \alpha(\mathrm{mAb} 343 \quad(1.8 \mathrm{mg} / \mathrm{ml})$ (curtsey of Wilbur FRanklin and Philip Low)), p53 (Ab-2, clone 1801; 1:500; Calbiochem, Cambridge, MA, USA; the antibody detects wild-type and mutated p53), p27 (Ab-2, clone DCS72; 1:500; Oncogene Research Products, Cambridge, MA, USA), Ki-67 (Mib-1; 1:50; Immunotech, Marseille, France), BCL2 (clone 124; 1:72; Dako, Glostrup, Denmark), thymidylate synthase (clone TS106/4H4B1, Zymed Laboratories, South San Francisco, CA, USA), MGMT (1:25; NeoMarkers, Fremont, CA, USA), MLH1 (clone G168-728, diluted 1:250, Pharmingen, San Diego, CA, USA) and MSH2 (clone FE11, diluted 1:50, Oncogene Research Products) were used. Samples were then incubated with biotinylated anti- mouse immunoglobulins at 1:500 dilution (Vector Laboratories, Burlingame, CA, USA) followed by avidin-biotin peroxidase complexes (1:25; Vector Laboratories) for $30 \mathrm{~min}$. Diaminobenzidine was used as the chromogen, and hematoxylin was used as the nuclear counterstain.

Cases with at least two of the three tissue cores showing analyzable staining were included in the final analysis. Those with only one or no cores with analyzable staining were regarded as tissue lost and not included in this study.

The FR $\alpha$ staining was evaluated using a previously described scoring system. ${ }^{22}$ Briefly, cases were scored as 0 when there was no staining in any of the cores, $1+$ when at least one core showing at least weak staining but not qualifying for $2+$; and $2+$ when at least two tissue cores showing moderate staining or one core showing strong staining. Examples of various staining intensities are illustrated in Figure 1.

For MGMT, MLH1 and MSH2 staining, 'abnormal' referred to loss of nuclear staining in $100 \%$ of tumor cells. For MLH1 and MSH2, cases that showed

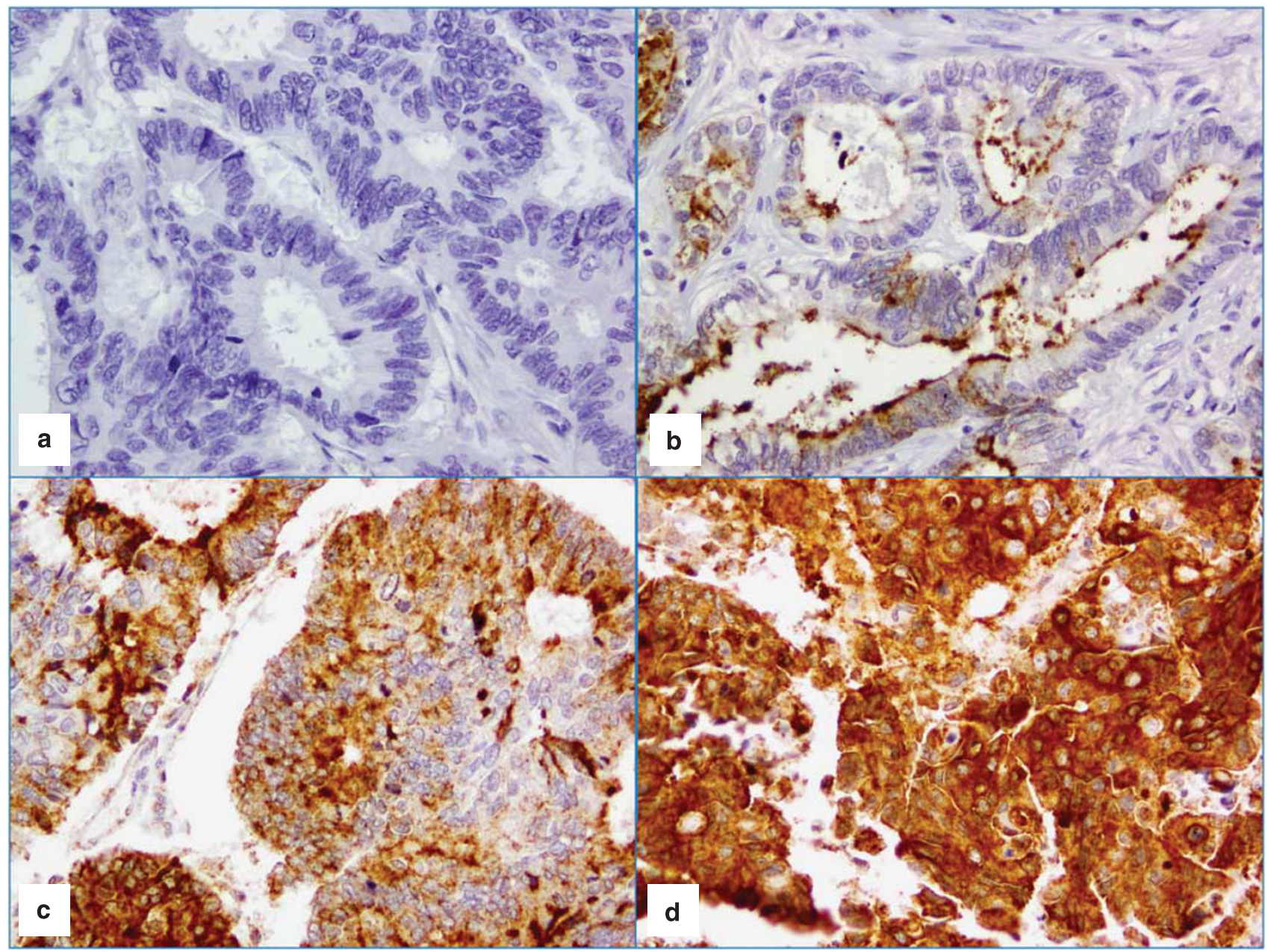

Figure 1 Examples of negative (a), weak (b), moderate (c) and strong (d) immunohistochemical staining for Folate Receptor- $\alpha$ in liver metastases from colorectal adenocarcinoma. 
abnormal staining (loss of nuclear staining in 100\% of tumor cells) or equivocal staining on tissue array sections were tested again on corresponding whole sections with MLH1 and PMS2 (clone A16-4, diluted 1:200, BD Pharmingen) or MSH2 and MSH6 (clone GRBP.P1/2.D4, diluted 1:200; Serotec, Raleigh, NC, USA), respectively. The findings of the whole sections represented the final data for these markers.

For all other markers, the positive staining was classified as continuum data (undetectable levels or $0 \%$ to homogeneous staining or $100 \%$ ). The cutoff values for tumor cell staining used in this study were defined as follows: (a) high Ki-67 proliferative index, if $>60 \%$ tumor cells stained positive; (b) p53 overexpression, if $>50 \%$ tumor cells stained positive; (c) BCL2 overexpression, if $>30 \%$ tumor cells stained positive; (d) p27 positivity, if $>20 \%$ tumor stained positive; and (e) thymidylate synthase overexpression, if $>30 \%$ tumor cells stained positive.

\section{Statistical Analysis}

Survival was measured from the time of liver resection to death or last follow-up and categorized as either early ( $\leq 2$ years) or late ( $>10$ years). All alive patients had at least 10 years of follow-up to avoid bias due to this dichotomization. Comparison of categorical variables was carried out using Fisher exact test, and continuous variables were presented as mean values and range and compared using a two-sample $t$ test. The association between FR $\alpha$ status and early death was adjusted for clinical risk score and positive margins using an exact logistic regression model. A $P$-value $<0.05$ was considered significant.

\section{Results}

\section{Clinical and Pathological Findings}

A total of 602 patients underwent liver resection at MSKCC for colorectal cancer liver metastases from 1985 to 1994. From this cohort, we identified 160 patients with sufficient pathological material; 56 belonging to the long-term survivor group and 104 to the early death group.

As summarized in Table 1, more patients in the early death group had multiple liver tumors (63 vs $43 \% ; P=0.04$ ), with a median number of two tumors compared with one in the long-term survivor group. The early death group was also more likely to have tumors greater than $5 \mathrm{~cm}(61 \mathrm{vs} 35 \% ; P<0.01)$. Median size of the largest tumor was $5.5 \mathrm{~cm}$ in early death group compared with $3.5 \mathrm{~cm}$ in the long-term survival group. Pre-hepatectomy CEA was more commonly greater than $200 \mathrm{ng} / \mathrm{ml}$ in the early death group (24 vs $7 \% ; P=0.01$ ). Median pre-hepatectomy CEA was $37.9 \mathrm{ng} / \mathrm{ml}$ in the early death group but only $8.5 \mathrm{ng} / \mathrm{ml}$ in long-term survivors. Clinical risk score greater than or equal to 3 was observed in $56 \%$ of the early death group compared with $16 \%$ of longterm survivors $(P<0.01)$. Median clinical risk score was 3 in the early death group and 2 in long-term survivors. No long-term survivors had positive resection margins.

\section{Immunohistochemical Findings}

The comparison of the results of immunohistochemical testing is summarized in Table 1. Analyzable data for FR $\alpha$ expression were obtained in 150 patients, of which $37(25 \%)$ were positive;

Table 1 Clinical, pathological and immunohistochemical characteristics in patients with hepatic colorectal cancer metastases and distinctly different survival

\begin{tabular}{|c|c|c|c|}
\hline & $\begin{array}{c}<2 \text {-year survival } \\
(\mathrm{n}=104)\end{array}$ & $\begin{array}{c}>10 \text {-year survival } \\
(\mathrm{n}=56)\end{array}$ & P-value \\
\hline Median age (range) & $62(28-82)$ & $62(32-79)$ & 0.88 \\
\hline Male & $71(68 \%)$ & $36(64 \%)$ & 0.61 \\
\hline Hemi-hepatectomy or more extensive & $70 / 104(67 \%)$ & $36 / 56(64 \%)$ & 0.70 \\
\hline Bilateral resection & $49 / 104(47 \%)$ & $15 / 56(27 \%)$ & 0.01 \\
\hline Disease-free interval $<12$ months & $50 / 103(49 \%)$ & $41 / 55(75 \%)$ & $<0.01$ \\
\hline Node-positive primary cancer & $65 / 96(68 \%)$ & $30 / 56(54 \%)$ & 0.08 \\
\hline More than one tumor in liver & $65 / 104(63 \%)$ & $25 / 55(46 \%)$ & 0.04 \\
\hline Largest tumor $>5 \mathrm{~cm}$ & $62 / 102(61 \%)$ & $19 / 54(35 \%)$ & $<0.01$ \\
\hline Pre-hepatectomy CEA > $200(\mathrm{ng} / \mathrm{ml})$ & $20 / 84(24 \%)$ & $4 / 55(7 \%)$ & 0.01 \\
\hline Margin positive & $21 / 104(20 \%)$ & $0 / 56$ & $<\mathbf{0 . 0 1}$ \\
\hline Clinical risk high score $(\geq 3)$ & $58 / 104(56 \%)$ & $9 / 56(16 \%)$ & $<\mathbf{0 . 0 1}$ \\
\hline FR $\alpha$ overexpression $(1+$ and $2+)$ & $30 / 95(32 \%)$ & $7 / 55(13 \%)$ & $\mathbf{0 . 0 3}$ \\
\hline \multicolumn{4}{|l|}{ P53 overexpression } \\
\hline (>50\% tumor positive) & $56 / 102(55 \%)$ & $23 / 56(41 \%)$ & 0.25 \\
\hline Ki-67 staining index $>60 \%$ & $51 / 102(50 \%)$ & $21 / 56(38 \%)$ & 0.13 \\
\hline P27 overexpression ( $>20 \%$ tumor positive) & $37 / 99(37 \%)$ & $21 / 55(38 \%)$ & 0.39 \\
\hline Thymidylate synthase overexpression ( $>20 \%$ tumor positive) & $45 / 101(45 \%)$ & $19 / 56(34 \%)$ & 0.42 \\
\hline BCL2 overexpression ( $>30 \%$ tumor positive) & $11 / 101(11 \%)$ & $8 / 56(15 \%)$ & 0.77 \\
\hline Loss of MGMT & $14 / 98(14 \%)$ & $13 / 56(23 \%)$ & 0.16 \\
\hline Loss of MLH1/MSH2 & $2 / 100(2 \%)$ & $4 / 55(7 \%)$ & 0.19 \\
\hline
\end{tabular}

Bold $P$ values $<0.05$ was considered significant. 
$22(15 \%)$ with a score of 1 and $15(10 \%)$ with a score of 2 . FR $\alpha$ expression was more commonly seen in tumors from the early death group (32 vs $13 \%$; $P=0.03)$. There were no other differences in expression of other tumor markers between the two groups.

\section{Correlation among Various Clinical and Pathological Features}

As listed in Table 2, there was no significant association between $\mathrm{FR} \alpha$ expression and patient demographics, tumor characteristics or immunohistochemical staining. The only statistically significant difference between these groups was a higher margin positive resection in $\mathrm{FR} \alpha$-positive patients (24 vs $10 \%$; $P=0.05)$. After excluding margin positive patients, $\mathrm{FR} \alpha$ continued to be more commonly expressed in the early death group compared with long-term survivors (28 vs 13\%; $P=0.05$ ).

Factors significantly associated with the longterm survivor group on univariatse analysis included $\mathrm{FR} \alpha$, disease-free interval, number of tumors, size of largest tumor and pre-hepatectomy CEA level (Table 1). As all of these variables (except for FR $\alpha$ ) are part of the clinical risk score and because our goal was to obtain adjusted estimates of the association between early death and FR $\alpha$ status rather than investigating prognostic factors, we used clinical risk score $(\geq 3$ vs $\leq 2)$ for an adjusted analysis. We also included positive margins, as this was the only variable that significantly differed between FR $\alpha$ positive and -negative patients. The results of this multivariate analysis are shown in Table 3. FR $\alpha$ remained a significant predictor of early death after adjusting for clinical risk score and margins. Patients who are $F R \alpha$ positive are at three-fold increased risk of early death, regardless of their clinical risk score or margins $(\mathrm{OR}=3.2,95 \%$ con- fidence interval: 1.1-10.3, $P=0.032$ ). To confirm this finding, we carried out a second analysis of FR $\alpha$ status and clinical risk score on the margin-negative patients only, and $\mathrm{FR} \alpha$ status retained its status as an independent predictor (data not shown).

\section{Discussion}

One of the objectives of this study was to analyze the expression pattern of $\mathrm{FR} \alpha$ in resected colorectal cancer liver metastases utilizing immunohistochemistry as the detection method. In keeping with what has been shown in a previous study, we found overexpression of $\mathrm{FR} \alpha$ in a subset of hepatic metastases. $^{21}$ Such information should provide a basis for further exploration of the utility of FR $\alpha$ targeted strategies in this patient population.

In this study, we found that $25 \%$ of resected colorectal cancer liver metastases demonstrated FR $\alpha$ expression; $10 \%$ with strong intensity. As our previous study that used the same detection method found a similar frequency in primary colorectal cancers $(33 \%, 9 \%$ with strong intensity, $n=177)$, it is tempting to assume that both the primary and metastatic tumors have a similar pattern of $\mathrm{FR} \alpha$ expression. However, as the study subjects in the current study were selected groups based on outcome, the frequency may not be representative of the

Table 3 Multivariate analysis for predictors of early death

\begin{tabular}{lccr}
\hline & Odds ratio & $95 \%$ CI & P-value \\
\hline FR $\alpha$ positive & 3.2 & $1.1,10.3$ & 0.032 \\
Clinical risk score $\geq 3$ & 6.2 & $2.5,17.1$ & $<0.001$ \\
Margin positive & 16.1 & 2.5, NA $^{\text {a }}$ & 0.001 \\
\hline
\end{tabular}

${ }^{a}$ All positive margin patients were in the early death group, hence the upper confidence limit cannot be estimated.

Table 2 FR $\alpha$ versus other clinical and pathological characteristics

\begin{tabular}{|c|c|c|c|}
\hline & $\begin{array}{l}\text { FR } \alpha \text { positive } \\
\quad(\mathrm{n}=37)\end{array}$ & $\begin{array}{c}F R \alpha \text { negative } \\
(\mathrm{n}=113)\end{array}$ & P-value \\
\hline Median age (range) & $61(33-79)$ & $63(28-82)$ & 0.34 \\
\hline Male/female & $28(76 \%)$ & $70(62 \%)$ & 0.13 \\
\hline Hemi-hepatectomy or more extensive & $23 / 37(62 \%)$ & $76 / 113(67 \%)$ & 0.57 \\
\hline Bilateral resection & $18 / 37(49 \%)$ & $41 / 113(36 \%)$ & 0.18 \\
\hline Disease-free interval $<12$ months & $20 / 37(54 \%)$ & $42 / 111(38 \%)$ & 0.08 \\
\hline Node-positive primary cancer & $21 / 31(68 \%)$ & $65 / 111(59 \%)$ & 0.36 \\
\hline More than one tumor in liver & $20 / 37(54 \%)$ & $62 / 112(55 \%)$ & 0.89 \\
\hline Largest tumor $>5 \mathrm{~cm}$ & $19 / 36(53 \%)$ & $58 / 111(52 \%)$ & 0.96 \\
\hline Pre-hepatectomy CEA > 200 (ng/ml) & $7 / 29(24 \%)$ & $15 / 101(15 \%)$ & 0.24 \\
\hline Margin positive & $9 / 37(24 \%)$ & $11 / 113(10 \%)$ & 0.05 \\
\hline Clinical risk high score $(\geq 3)$ & $16 / 37(43 \%)$ & $45 / 113(40 \%)$ & 0.71 \\
\hline P53 overexpression ( $>50 \%$ tumor positive) & $27 / 37(73 \%)$ & $70 / 112(63 \%)$ & 0.32 \\
\hline Ki-67 staining index $>60 \%$ & $20 / 37(54 \%)$ & $47 / 112(42 \%)$ & 0.20 \\
\hline P27 overexpression ( $>20 \%$ tumor positive) & $30 / 37(81 \%)$ & 78/107 (73\%) & 0.38 \\
\hline Thymidylate synthase overexpression ( $>20 \%$ tumor positive) & $20 / 37(54 \%)$ & $40 / 111(36 \%)$ & 0.08 \\
\hline BCL2 overexpression ( $>30 \%$ tumor positive) & $5 / 37(14 \%)$ & $13 / 111(12 \%)$ & 0.78 \\
\hline Loss of MGMT & $3 / 37(8 \%)$ & $22 / 109(20 \%)$ & 0.13 \\
\hline Loss of MLH1/MSH2 & $0 / 37(0 \%)$ & $6 / 110(5 \%)$ & 0.34 \\
\hline
\end{tabular}

Bold $P$ values $<0.05$ was considered significant. 
general population of patients with advanced colorectal cancer. Studies of paired primary and metastatic colorectal cancers from the same patients would be needed to answer whether FR $\alpha$ expression in colorectal cancers persists as a tumor progresses in an individual patient. Such data may become relevant should FR $\alpha$-targeted therapies find utility in colorectal cancer patients.

The second purpose of this study was to evaluate the potential prognostic significance of FR $\alpha$ expression in resected colorectal cancer liver metastases in comparison with other commonly evaluated molecular markers. Although none of the common molecular markers were associated with early death or 10-year survival, FR $\alpha$ overexpression independently associated with early death in addition to other previously defined clinical risk factors such as bilateral resection, disease-free interval, number of tumor nodules, size of the largest tumor, pre-hepatectomy CEA level and positive margin. The significance of $\mathrm{FR} \alpha$ was maintained on multivariate analysis and had added predictive value to our best clinical predictors of outcome including the clinical risk score.

The molecular markers we assessed along with FR $\alpha$ included p53, p27, BCL2, ki67, MLH1, MSH2, MGMT and thymidylate synthase. These and other related markers have been extensively studied in recent years in primary colorectal cancers. ${ }^{4,22-29}$ The most frequent scenario, in which these markers were assessed has been stage II cancers to identify patients who might benefit from adjuvant therapy but would otherwise be denied of such an opportunity. ${ }^{30}$ The data thus far have been inconclusive. Although none of the markers has yet reached sufficient statistical significance to allow routine clinical use, microsatellite instability holds great promise as a favorable prognostic marker that may find routine use in clinic practice in the foreseeable future. In this study, we hoped to evaluate the prognostic significance of these markers in resected stage-IV colorectal cancer patients, a scenario that is not well covered in the literature but is becoming increasingly pertinent as treatment paradigms include surgical resection as a standard component.

Our data suggest that the expression of the molecular markers p53, p27, BCL2, ki-67 and thymidylate synthase do not have sufficient statistical significance as prognostic markers in resected colorectal cancer liver metastases. With regard to microsatellite instability-related markers, we found only six tumors that demonstrated loss of either MLH1 or MSH2 by immunohistochemistry in 155 metastases (4\%). This low rate is in keeping with the literature, indicating a significantly lower frequency of microsatellite instability-high phenotype in advanced colorectal cancers when compared with primary cancers, and likely reflecting the fact that microsatellite instable cancers are less likely to metastasize. ${ }^{28}$ The low number of positive cases in our series makes it impossible to draw any mean- ingful conclusion about its prognostic significance in patients with resected colorectal cancer liver metastases. Thymidylate synthase has been studied in resected colorectal cancer liver metastases with mixed results. One study utilizing immunohistochemistry demonstrated thymidylate synthase to be associated with outcome, but this effect was abrogated by the use of adjuvant hepatic arterial infusion chemotherapy. ${ }^{31}$ Another study analyzed TS expression by reverse-transcription PCR and found that TS was not associated with outcome. ${ }^{32}$

Immunohistochemical detection of MGMT has been used as a surrogate marker for the CpG island methylator phenotype (CIMP) in colorectal cancers, and has been shown to correlate closely with results obtained by using methylation-specific PCR assays. $^{28,33}$ Data regarding loss of MGMT using immunohistochemistry in colorectal metastasis are largely lacking. Our analysis in this study demonstrated a trend towards more frequent loss of MGMT (implying increased frequency of CIMP) in patients with long-term survival; however, this trend fell short of statistical significance.

In contrast to the aforementioned markers, FR $\alpha$ expression was found to be significantly associated with early death after resection of colorectal cancer liver metastases on both univariate and multivariate analyses. Importantly, this was demonstrated in the context of many well-defined clinical risk factors. Furthermore, the addition of $\mathrm{FR} \alpha$ status to the clinical risk score may strengthen prognostication. Such an observation is in line with our previous findings in primary colorectal cancers, as well as that observed in ovarian, breast and endometrial carcinoma. ${ }^{9-11}$ In all of these tumor types including both primary and metastatic colorectal cancer, overexpression of FR $\alpha$ detected by immunohistochemistry had either a strong trend towards, or significantly correlated with a worse survival. Interestingly, however, a study that evaluated 119 non-small cell lung cancers found high FR $\alpha$ expression to be correlated with a better survival. ${ }^{12}$ Nonetheless, the association of $\mathrm{FR} \alpha$ expression with outcome should promote further research efforts on the prognostic significance of this marker in larger more heterogeneous colorectal cancer populations.

The potential prognostic significance of $F R \alpha$ in various cancers is conceptually conceivable as folate has a key role in DNA synthesis and methylation. ${ }^{34}$ It is of interest to note that all six tumors that had abnormal DNA mismatch repair protein expression (implying microsatellite instability-high phenotype) in our series were from the FR $\alpha$-negative group. Although the microsatellite instability phenotype and FR $\alpha$ expression were not statistically associated because of a small number of events, this tendency of microsatellite instability-high to occur in FR $\alpha$ negative tumors was observed in our previous study of primary colorectal cancers. ${ }^{21}$ It seems possible that both the association between microsatellite 
instability-high and better outcome (as has been observed in primary cancers), and the association between the lack of FR $\alpha$ expression and better outcome may be inter-related. Further investigation in this regard may help reveal new mechanisms that interconnect these events in colorectal cancer development and/or progression.

In conclusion, this study demonstrates that FR $\alpha$ expression is present in a subset of resected colorectal cancer liver metastases, and is independently associated with early death after hepatic resection. Studies on the use of FR $\alpha$-targeted strategies in resectable stage-IV colorectal cancer patients and on evaluation of $\mathrm{FR} \alpha$ as a cancer prognostic marker are justified.

\section{Disclosure/conflict of interest}

The authors declare no conflict of interest.

\section{References}

1 Fong Y, Fortner J, Sun RL, et al. Clinical score for predicting recurrence after hepatic resection for metastatic colorectal cancer: analysis of 1001 consecutive cases. Ann Surg 1999;230:309-318; discussion 18-21.

2 Rees M, Tekkis PP, Welsh FK, et al. Evaluation of longterm survival after hepatic resection for metastatic colorectal cancer: a multifactorial model of 929 patients. Ann Surg 2008;247:125-135.

3 Abdalla EK, Adam R, Bilchik AJ, et al. Improving resectability of hepatic colorectal metastases: expert consensus statement. Ann Surg Oncol 2006;13: 1271-1280.

4 Walther A, Johnstone E, Swanton C, et al. Genetic prognostic and predictive markers in colorectal cancer. Nat Rev Cancer 2009;9:489-499.

5 Basal E, Eghbali-Fatourechi GZ, Kalli KR, et al. Functional folate receptor alpha is elevated in the blood of ovarian cancer patients. PLoS One 2009;4:e6292.

6 Henderson GB. Folate-binding proteins. Annu Rev Nutr 1990;10:319-335.

7 Xia W, Low PS. Folate-targeted therapies for cancer. J Med Chem 2010;53:6811-6824.

8 Kularatne SA, Low PS. Targeting of nanoparticles: folate receptor. Methods Mol Biol 2010;624:249-265.

9 Brown Jones M, Neuper C, Clayton A, et al. Rationale for folate receptor alpha targeted therapy in 'high risk' endometrial carcinomas. Int J Cancer 2008;123: 1699-1703.

10 Hartmann LC, Keeney GL, Lingle WL, et al. Folate receptor overexpression is associated with poor outcome in breast cancer. Int J Cancer 2007;121:938-942.

11 Toffoli G, Russo A, Gallo A, et al. Expression of folate binding protein as a prognostic factor for response to platinum-containing chemotherapy and survival in human ovarian cancer. Int J Cancer 1998;79:121-126.

12 Iwakiri S, Sonobe M, Nagai S, et al. Expression status of folate receptor alpha is significantly correlated with prognosis in non-small-cell lung cancers. Ann Surg Oncol 2008;15:889-899.

13 Parker N, Turk MJ, Westrick E, et al. Folate receptor expression in carcinomas and normal tissues deter- mined by a quantitative radioligand binding assay. Anal Biochem 2005;338:284-293.

14 Low PS, Antony AC. Folate receptor-targeted drugs for cancer and inflammatory diseases. Adv Drug Deliv Rev 2004;56:1055-1058.

15 Lu Y, Sega E, Leamon CP, et al. Folate receptor-targeted immunotherapy of cancer: mechanism and therapeutic potential. Adv Drug Deliv Rev 2004;56: 1161-1176.

16 Leamon CP, Reddy JA, Vlahov IR, et al Preclinical antitumor activity of a novel folate-targeted dual drug conjugate. Mol Pharm 2007;4:659-667.

17 Li J, Sausville EA, Klein PJ, et al. Clinical pharmacokinetics and exposure-toxicity relationship of a folateVinca alkaloid conjugate EC145 in cancer patients. J Clin Pharmacol 2009;49:1467-1476.

18 Reddy JA, Dorton R, Westrick E, et al. Preclinical evaluation of EC145, a folate-vinca alkaloid conjugate. Cancer Res 2007;67:4434-4442.

19 Siegel BA, Dehdashti F, Mutch DG, et al. Evaluation of 111In-DTPA-folate as a receptor-targeted diagnostic agent for ovarian cancer: initial clinical results. J Nucl Med 2003;44:700-707.

20 Naumann RW, Symanowski JT, Ghamande SA, et al. PRECEDENT: A randomized phase II trial comparing EC145 and pegylated liposomal doxorubicin (PLD) in combination, versus PLD alone, in subjects with platinum-resistant ovarian cancer. Asco Meeting Abstr 2010;28:LBA5012B-LBA5LBAb.

21 Shia J, Klimstra DS, Nitzkorski JR, et al. Immunohistochemical expression of folate receptor alpha in colorectal carcinoma: patterns and biological significance. Hum Pathol 2008;39:498-505.

22 Moore HG, Shia J, Klimstra DS, et al Expression of p27 in residual rectal cancer after preoperative chemoradiation predicts long-term outcome. Ann Surg Oncol 2004;11:955-961.

23 Munro AJ, Lain S, Lane DP. P53 abnormalities and outcomes in colorectal cancer: a systematic review. Br J Cancer 2005;92:434-444.

24 Ogino S, Nosho K, Kirkner GJ, et al. CpG island methylator phenotype, microsatellite instability, BRAF mutation and clinical outcome in colon cancer. Gut 2009;58:90-96.

25 Popat S, Hubner R, Houlston RS. Systematic review of microsatellite instability and colorectal cancer prognosis. J Clin Oncol 2005;23:609-618.

26 Popat S, Matakidou A, Houlston RS. Thymidylate synthase expression and prognosis in colorectal cancer: a systematic review and meta-analysis. J Clin Oncol 2004;22:529-536.

27 Rosati G, Chiacchio R, Reggiardo G, et al. Thymidylate synthase expression, p53, bcl-2, Ki-67 and p27 in colorectal cancer: relationships with tumor recurrence and survival. Tumour Biol 2004;25:258-263.

28 Russo A, Bazan V, Iacopetta B, et al. The TP53 colorectal cancer international collaborative study on the prognostic and predictive significance of p53 mutation: influence of tumor site, type of mutation, and adjuvant treatment. J Clin Oncol 2005;23: $7518-7528$.

29 Shen L, Toyota M, Kondo Y, et al. Integrated genetic and epigenetic analysis identifies three different subclasses of colon cancer. Proc Natl Acad Sci USA 2007;104:18654-18659.

30 Shankaran V, Wisinski KB, Mulcahy MF, et al. The role of molecular markers in predicting response to therapy 
in patients with colorectal cancer. Mol Diagn Ther 2008;12:87-98.

31 Gonen M, Hummer A, Zervoudakis A, et al. Thymidylate synthase expression in hepatic tumors is a predictor of survival and progression in patients with resectable metastatic colorectal cancer. J Clin Oncol 2003;21:406-412.

32 Lassmann S, Tang L, Capanu M, et al. Predictive molecular markers for colorectal cancer patients with resected liver metastasis and adjuvant chemotherapy. Gastroenterology 2007;133:1831-1839.

33 Mikami T, Yoshida T, Numata Y, et al. Low frequency of promoter methylation of O6-methylguanine DNA methyltransferase and hMLH1 in ulcerative colitisassociated tumors: comparison with sporadic colonic tumors. Am J Clin Pathol 2007;127:366-373.

34 Choi SW, Mason JB. Folate and carcinogenesis: an integrated scheme. J Nutr 2000;130:129-132. 